\title{
Nonextensive statistical mechanics: Some links with astronomical phenomena *
}

\author{
Constantino Tsallis \\ Centro Brasileiro de Pesquisas Físicas \\ Rua Xavier Sigaud 150, 22290-180 Rio de Janeiro, RJ, Brazil \\ Domingo Prato \\ Facultad de Matematica, Astronomia y Fisica, Universidad Nacional de Cordoba, Argentina
}

\author{
Angel R. Plastino ${ }^{\dagger}$ \\ Facultad de Ciencias Astronomicas y Geofisicas \\ Universidad Nacional de La Plata and CONICET, CC 727, 1900 La Plata, Argentina \\ and \\ Departament de Fisica, Universitat de les Illes Balears, 07122, Palma de Mallorca, Spain
}

(November 29, 2018)

\begin{abstract}
A variety of astronomical phenomena appear to not satisfy the ergodic hypothesis in the relevant stationary state, if any. As such, there is no reason
\end{abstract}

\footnotetext{
*To appear in the Proceedings of the XIth United Nations / European Space Agency Workshop on Basic Space Sciences, Office for Outer Space Affairs / United Nations (Cordoba, 9-13 September 2002), ed. H. Haubold, special issue of Astrophysics and Space Science (Kluwer Academic Publishers, Dordrecht, 2003).

†tsallis@cbpf.br, prato@mail.famaf.unc.edu.ar, vdfsarp9@clust.uib.es
} 
for expecting the applicability of Boltzmann-Gibbs (BG) statistical mechanics. Some of these phenomena appear to follow, instead, nonextensive statistical mechanics. In the same manner that the BG formalism is based on the entropy $S_{B G}=-k \sum_{i} p_{i} \ln p_{i}$, the nonextensive one is based on the form $S_{q}=k\left(1-\sum_{i} p_{i}^{q}\right) /(q-1)\left(\right.$ with $\left.S_{1}=S_{B G}\right)$. The stationary states of the former are characterized by an exponential dependence on the energy, whereas those of the latter are characterized by an (asymptotic) power-law. A brief review of this theory is given here, as well as of some of its applications, such as the solar neutrino problem, polytropic self-gravitating systems, galactic peculiar velocities, cosmic rays and some cosmological aspects. In addition to these, an analogy with the Keplerian elliptic orbits versus the Ptolemaic epicycles is developed, where we show that optimizing $S_{q}$ with a few constraints is equivalent to optimizing $S_{B G}$ with an infinite number of constraints.

\section{INTRODUCTION}

Connections between dynamics and thermodynamics are far from being completely clarified. For instance, long-range interactions are expected to substantially modify various usual thermodynamical properties. E. Fermi addressed such question with the following words [1]:

The entropy of a system composed of several parts is very often equal to the sum of the entropies of all the parts. This is true if the energy of the system is the sum of the energies of all the parts and if the work performed by the system during a transformation is equal to the sum of the amounts of work performed by all the parts. Notice that these conditions are not quite obvious and that in some cases they may not be fulfilled. Thus, for example, in the case of a system composed of two homogeneous substances, it will be possible to express the energy as the sum of the energies of the two substances only if we can neglect the surface energy of the two substances where they are in contact. The surface energy can generally be neglected only if the two substances are not very finely subdivided; otherwise, it can play a 
considerable role.

In those of L. Tisza [2]:

The situation is different for the additivity postulate $P$ a2, the validity of which cannot be inferred from general principles. We have to require that the interaction energy between thermodynamic systems be negligible. This assumption is closely related to the homogeneity postulate $P$ d1. From the molecular point of view, additivity and homogeneity can be expected to be reasonable approximations for systems containing many particles, provided that the intramolecular forces have a short range character.

Finally, in those of P.T. Landsberg [3]:

The presence of long-range forces causes important amendments to thermodynamics, some of which are not fully investigated as yet.

In recent papers, also E.G.D. Cohen [4] and M. Baranger [5] have addressed this question. Indeed, a significant amount of systems, e.g., turbulent fluids ( $[6,7]$ and references therein), electron-positron annihilation [8], quark-gluon plasma [9], economics [10-12], motion of Hydra viridissima [13], kinetic theory [14], classical chaos [15], quantum chaos [16], quantum entanglement [17], anomalous diffusion [18], long-range-interacting many-body classical Hamiltonian systems ( [19] and references therein), internet dynamics [20], and others, are known nowadays which in no trivial way accomodate within BG statistical mechanical concepts. Systems like these have been handled with the functions and concepts which naturally emerge within nonextensive statistical mechanics [21-23].

The basic building block of nonextensive statistical mechanics is the nonextensive entropy $[21]$

$$
S_{q} \equiv k \frac{1-\sum_{i=1}^{W} p_{i}^{q}}{q-1} \quad(q \in \mathcal{R})
$$

The entropic index $q$ characterizes the statistics we are dealing with; $q=1$ recovers the usual BG expression

$$
S_{1}=S_{B G}=-k \sum_{i=1}^{W} p_{i} \ln p_{i} .
$$


We may think of $q$ as a biasing parameter: $q<1$ privileges rare events, while $q>1$ privileges common events. Indeed, $p<1$ raised to a power $q<1$ yields a value larger than $p$, and the relative increase $p^{q} / p=p^{q-1}$ is a decreasing function of $p$, i.e., values of $p$ closer to 0 (rare events) are benefited. Correspondingly, for $q>1$, values of $p$ closer to 1 (common events) are privileged. Therefore, the BG theory (i.e., $q=1$ ) is the unbiased statistics. A concrete consequence of this is that the BG formalism yields exponential equilibrium distributions (and time behavior of typical relaxation functions), whereas nonextensive statistics yields (asymptotic) power-law distributions (and relaxation functions). Since the BG exponential is recovered as a limiting case, we are talking of a generalization, not an alternative.

To obtain the probability distribution associated with the relevant stationary state (thermal equilibrium or metaequilibrium) of our system we must optimize the entropic form (1) under the following constraints $[21,22]$ :

$$
\sum_{i} p_{i}=1
$$

and

$$
\frac{\sum_{i} p_{i}^{q} E_{i}}{\sum_{i} p_{i}^{q}}=U_{q},
$$

where $\left\{E_{i}\right\}$ is the set of eigenvalues of the Hamiltonian (with specific boundary conditions), and $U_{q}$ is a fixed and finite number. This optimization yields

$$
p_{i}=\frac{\left[1-(1-q) \beta_{q}\left(E_{i}-U_{q}\right)\right]^{1 /(1-q)}}{Z_{q}},
$$

where

$$
Z_{q} \equiv \sum_{j}\left[1-(1-q) \beta_{q}\left(E_{j}-U_{q}\right)\right]^{1 /(1-q)}
$$

and

$$
\beta_{q} \equiv \frac{\beta}{\sum_{j} p_{j}^{q}},
$$

$\beta$ being the optimization Lagrange parameter associated with the generalized internal energy $U_{q}$. Equation (5) can be rewritten as 


$$
p_{i} \propto\left[1-(1-q) \beta^{\prime} E_{i}\right]^{1 /(1-q)} \equiv e_{q}^{-\beta^{\prime} E_{i}}
$$

where $\beta^{\prime}$ is a renormalized inverse "temperature", and the $q$-exponential function is defined as $e_{q}^{x} \equiv[1+(1-q) x]^{1 /(1-q)}=1 /[1-(q-1) x]^{1 /(q-1)}\left(\right.$ with $\left.e_{1}^{x}=e^{x}\right)$. This function replaces, in a vast number of relations and phenomena, the usual BG factor. In particular, the ubiquitous

Gaussian distribution $\propto e^{-a x^{2}}$ becomes generalized into the distribution $\propto e_{q}^{-a_{q} x^{2}}=1 /[1+$ $\left.(q-1) a_{q} x^{2}\right]^{1 /(q-1)}$ (fat-tailed if $q>1$, and with a cutoff if $\left.q<1\right)$.

\section{ANALOGY WITH THE PTOLEMY-KEPLER PROBLEM}

Ancient Greeks believed, through philosophical arguments, that the orbit of any celestial body ought to be a "perfect" geometrical figure, namely a circle. This belief was very consistent with the observations of the motion of the stars. Not so, however, with the observations of the motion of the planets (a word whose etymology precisely is wanderer). This nontrivial problem was attacked by Ptolemy. He introduced, with sensible success at that time, the idea that the motion of a planet would be that of a circle rotating around another circle, in turn rotating around another circle, and so on. The idea of such epicycles was recursively used by most astronomers along the centuries over large regions of the civilized world [24]. Before Kepler's time, some astronomers had used up to several dozens(!) of epicycles one on top of the other, in order to increase the precision of their calculations. Then Kepler arrived and proposed that the orbit of a planet around the sun would be that of a geometrical figure which generalizes the circle, namely that of an ellipse. This figure needs, for being characterized, one new parameter, namely the eccentricity $\epsilon$, in addition to say its largest diameter. The circle is thus recovered as the particular case $\epsilon=0$. In very few years, practically all the astronomers of Europe abandoned the cumbersome calculations with the Ptolemaic epicycles, and adopted the Keplerian ellipses. Ptolemy idea was nevertheless not wrong at all! Indeed, the planetary elliptic motion can be described in terms of infinite series, whose terms can be identified with the Ptolemaic epicycles. In other words, the elliptic keplerian motion can be regarded as resulting from an "infinite series of epicycles". 
A very lucid discussion of the correspondence between (i) the series expansion of the elliptic motion and (ii) the Ptolemaic geometric construction, was provided by Hoyle [25]. The alluded series for the planetary elliptic motion can be found in any text-book of Celestial Mechanics [26], and are given (in polar coordinates, as a function of time $t$ ) by

$$
\theta=n t+2 \epsilon \sin n t+\frac{5}{4} \epsilon^{2} \sin 2 n t+\ldots
$$

and

$$
\frac{r}{a}=1-\epsilon \cos n t+\frac{1}{2} \epsilon^{2}(1-\cos 2 n t)+\ldots
$$

where $a$ is the semi-major diameter, and $n \equiv 2 \pi / P, P$ being the so called sidereal period. If the orbit is a circle, i.e., $\epsilon=0$, then each polar coordinate has only one term in its expansion. For any value of $\epsilon \neq 0$, an infinite number of terms must be used if one wants the exact Keplerian answer. This sudden change in the number of terms of course reflects the equally sudden change of symmetry when one goes from the circle to the ellipse. In other words, if one wants to stick to circles, that is perfectly possible and correct, although the price to be paid is that one has to consider an infinite number of circles. The other, simpler, possibility, is of course to use only one ellipse. One has however to know, through any accessible procedure, the value of $\epsilon$ for that particular planet.

Let us go back now to the problem of the use and status of the nonextensive entropy $S_{q}$ as given in Eq. (1). How universal is the BG entropy? How $S_{q}$ fundamentally relates to it? What is the status of the entropic index $q$, which characterizes universality classes of nonextensivity? (The most famous one being of course the $q=1$ universality class). Let us address this epistemologically interesting point through the canonical ensemble associated with conservative Hamiltonian systems.

Let us first develop the q-logarithm function

$$
\ln _{q} x \equiv \frac{x^{1-q}-1}{1-q}=\frac{e^{(1-q) \ln x}-1}{1-q}=\ln x-\frac{q-1}{2} \ln ^{2} x+\frac{(q-1)^{2}}{6} \ln ^{3} x+\ldots
$$

as well as 


$$
p_{i}^{q-1}=e^{(q-1) \ln p_{i}}=1+(q-1) \ln p_{i}+\frac{(q-1)^{2}}{2} \ln ^{2} p_{i}+\ldots
$$

The functional to be optimized for the canonical ensemble is

$$
\Phi_{q}\left(\left\{p_{i}\right\}\right) \equiv \frac{S_{q}\left(\left\{p_{i}\right\}\right)}{k}+\alpha \sum_{i} p_{i}+\beta \frac{\sum_{i} p_{i}^{q} E_{i}}{\sum_{i} p_{i}^{q}}=\left\langle\ln _{q}\left(1 / p_{i}\right)\right\rangle+\alpha \sum_{i} p_{i}+\beta \frac{\left\langle p_{i}^{q-1} E_{i}\right\rangle}{\left\langle p_{i}^{q-1}\right\rangle},
$$

where $\alpha$ and $\beta$ are Lagrange parameters, and $\langle\ldots\rangle \equiv \sum_{i} p_{i}(\ldots)$. We use now the series $(11)$ and (12) inside Eq. (13), and obtain

$$
\begin{array}{r}
\Phi_{q}\left(\left\{p_{i}\right\}\right)=\frac{S_{B G}\left(\left\{p_{i}\right\}\right)}{k}-\frac{q-1}{2}\left\langle\ln ^{2} p_{i}\right\rangle-\frac{(q-1)^{2}}{6}\left\langle\ln ^{3} p_{i}\right\rangle+\ldots+\alpha \sum_{i} p_{i} \\
+\beta\left\langle E_{i}\right\rangle+\beta(q-1)\left[\left\langle E_{i} \ln p_{i}\right\rangle-\left\langle E_{i}\right\rangle\left\langle\ln p_{i}\right\rangle\right] \\
+\beta \frac{(q-1)^{2}}{2}\left[\left\langle E_{i} \ln ^{2} p_{i}\right\rangle-\left\langle E_{i}\right\rangle\left\langle\ln ^{2} p_{i}\right\rangle+2\left\langle E_{i}\right\rangle\left\langle\ln p_{i}\right\rangle^{2}-2\left\langle E_{i} \ln p_{i}\right\rangle\left\langle\ln p_{i}\right\rangle\right]+\ldots \\
=\Phi_{1}\left(\left\{p_{i}\right\}\right) \\
-\frac{q-1}{2}\left\langle\ln ^{2} p_{i}\right\rangle-\frac{(q-1)^{2}}{6}\left\langle\ln ^{3} p_{i}\right\rangle+\ldots+\beta(q-1)\left[\left\langle E_{i} \ln p_{i}\right\rangle-\left\langle E_{i}\right\rangle\left\langle\ln p_{i}\right\rangle\right] \\
+\beta \frac{(q-1)^{2}}{2}\left[\left\langle E_{i} \ln ^{2} p_{i}\right\rangle-\left\langle E_{i}\right\rangle\left\langle\ln ^{2} p_{i}\right\rangle+2\left\langle E_{i}\right\rangle\left\langle\ln p_{i}\right\rangle^{2}-2\left\langle E_{i} \ln p_{i}\right\rangle\left\langle\ln p_{i}\right\rangle\right]+\ldots
\end{array}
$$

This optimization is the usual one within BG statistical mechanics if $q=1$, having a few constraints. It suddenly becomes a quite complex one, with an infinite number of constraints, if $q \neq 1$. Let us further analyze the generic case.

Let us consider the following generic optimization problem on the basis of the $S_{B G}$ entropy. The constraints to be used are Eq. (3), as well as the following ones:

$$
\left\langle\ln ^{k} p\right\rangle=C_{k}(k=2,3,4, \ldots),
$$

where $\left\{C_{k}\right\}$ are fixed finite quantities, to which we shall associate the Lagrange parameters $\left\{\gamma_{k}\right\}$. In addition to these constraints, we also have the following ones:

$$
\begin{gathered}
\left\langle E_{i}\right\rangle=D_{1}, \\
\left\langle E_{i} \ln p_{i}\right\rangle-\left\langle E_{i}\right\rangle\left\langle\ln p_{i}\right\rangle=\left\langle\left(E_{i}-\left\langle E_{i}\right\rangle\right)\left(\ln p_{i}-\left\langle\ln p_{i}\right\rangle\right)\right\rangle=D_{2}, \\
\left\langle E_{i} \ln ^{2} p_{i}\right\rangle-\left\langle E_{i}\right\rangle\left\langle\ln ^{2} p_{i}\right\rangle+2\left\langle E_{i}\right\rangle\left\langle\ln p_{i}\right\rangle^{2}-2\left\langle E_{i} \ln p_{i}\right\rangle\left\langle\ln p_{i}\right\rangle=D_{3},
\end{gathered}
$$


etc, where $D_{1}, D_{2}, D_{3}, \ldots, D_{l}, \ldots$ are fixed finite quantities, to which we shall associate the Lagrange parameters $\delta_{1}, \delta_{2}, \delta_{3}, \ldots, \delta_{l}, \ldots$. The optimization of $S_{B G}$ with all these constraints provides an optimizing distribution noted $p\left(E_{i} ; \alpha,\left\{\gamma_{k}\right\},\left\{\delta_{l}\right\}\right)$. We shall next consider a particular case of this distribution, the one where we impose

$$
\begin{gathered}
\gamma_{k}=-\frac{(q-1)^{k-1}}{k !} \quad(k=2,3,4, \ldots), \\
\delta_{1}=\beta, \\
\delta_{2}=\beta(q-1), \\
\delta_{3}=\beta \frac{(q-1)^{2}}{2},
\end{gathered}
$$

etc. Let us stress that taking a particular case of $p\left(E_{i} ; \alpha,\left\{\gamma_{k}\right\},\left\{\delta_{l}\right\}\right)$ is perfectly legitimate. [Indeed, as an illustration, let us remind that in the usual BG statistical mechanics, the distribution corresponding to the microcanonical ensemble can be viewed as the $\beta=0$ particular case of the distribution associated with the canonical ensemble]. Consequently the stationary distribution becomes a function only of $\left(E_{i}, \beta, q\right)$. We may alternatively consider that we have defined a specific new ensemble, whose distribution is fully known once we know $\beta$ and $q$. The inverse "temperature" $\beta$ depends on the physical thermostat with which the system is in contact, and $q$ depends on the microscopic dynamics of the specific physical system. The symmetry change that has occurred from $q=1$ to $q \neq 1$ hopefully corresponds to the fact that ergodicity (homogeneity in phase space) has been lost, and occupancy is now related to a presumably (multi)fractal or hierarchical geometry in phase space. The fact that only one new parameter emerges (namely $q$ ), and not the infinite number that would correspond to the entire multifractal function $f(\alpha)$, should be presumably related to the expectation that the thermodynamical properties would basically depend only on one number, namely the Hausdorff dimension of the system (see [27] for a connection between $q$ and the Hausdorff dimension for the simple case of the logistic-like 
maps). Although further studies are necessary to transparently establish the connection of the geometry in phase space with $q$, we have shown herein that indeed the optimization of $S_{q}$ with a few constraints is equivalent to the optimization of $S_{B G}$ with an infinite number of them. Therefore, it is in principle possible and correct to use $S_{B G}$ for such complex systems. The price to be paid is the fact that an infinite number of constraints must be, in one way or another, taken into account. The alternative is to use $S_{q}$ with just a few constraints, but one needs to know the value for $q$ to be used. The analogy with the Ptolemy-Kepler problem, where $\epsilon$ must be known, is therefore, in our opinion, quite striking.

\section{APPLICATIONS}

Let us now briefly review five recent applications of the ideas associated with nonextensive statistical mechanics to phenomena in astronomy and astrophysics, namely the solar neutrino deficit [28], self-gravitating polytropic systems [29-31], peculiar velocities of galaxy clusters [37], the flux of cosmic rays [38], and some cosmological effects [39].

\section{Solar neutrino problem:}

The solar plasma is believed to produce large amounts of neutrinos through a variety of mechanisms (e.g., the proton-proton chain). The calculation done using the so called Solar Standard Model (SSM) results in a neutrino flux over the Earth, which is roughly the double of what is measured. This is sometimes referred to as the neutrino problem or the neutrino enigma. There is by no means proof that this neutrino flux defect is due to a single cause. It has recently been verified that neutrino oscillations do seem to exist, which would account for part of the deficit. But it is not at all clear that it would account for the entire discrepancy. Quarati and collaborators [28] argue that part of it - even, perhaps, an appreciable part of it - could be due to the fact that BG statistical mechanics is used within the SSM. The solar plasma involves turbulence, long-range interactions, possibly long-range memory processes, all of them phenomena that could easily defy the applicability of the BG formalism. Then they show [28] in great detail how the modification of the "tail" of the energy distribution 
could considerably modify the neutrino flux to be expected. Consequently, small departures from $q=1$ (e.g., $|q-1|$ of the order of 0.1 or even less) would be enough to produce as much as $50 \%$ difference in the flux. This is due to the fact that most of the neutrino flux occurs at what is called the Gamow peak. This peak occurs at energies quite above the temperature (say 10 times larger), i.e., at energies in the tail of the distribution.

\section{Polytropic Equilibrium Solutions to the Vlasov-Poisson Equations:}

The first physical application of the non-extensive thermostatistical formalism was related to the study of maximum entropy solutions to the Vlasov-Poisson equations describing self gravitating $N$-body systems like galaxies $[29,30]$. The maximization of the standard Boltzmann-Gibbs entropy under the constraints imposed by mass and energy conservation lead to the isothermal sphere distribution, which has infinite mass and energy [32]. In [29,30], it was shown that the extremalization of the non extensive $q$-entropy under the same constraints leads to the stellar polytropic sphere distributions which, for a certain range of the $q$ parameter, are endowed with finite mass and energy, as physically expected. This constituted the first clue suggesting that the generalized thermostatistical formalism based on $S_{q}$ may be of some relevance for the study of systems exhibiting non extensive thermodynamical properties due to long range interactions. The possible usefulness of non-logarithmic entropic measures in the study of stellar systems had also been suggested in [33].

Stellar polytropic sphere distributions are of the form

$$
\begin{array}{rlrl}
f(\mathbf{x}, \mathbf{v})=f(\tilde{\epsilon}) & =A\left(\Phi_{0}-\tilde{\epsilon}\right)^{n-3 / 2} & & \tilde{\epsilon} \leq \Phi_{0} \\
& =\quad 0 & 0 & \tilde{\epsilon}>\Phi_{0},
\end{array}
$$

where

$$
\tilde{\epsilon}=\frac{1}{2} \mathbf{v}^{2}+\Phi(\mathbf{x})
$$

is the total energy (per unit mass) of an individual star, and $A, \Phi_{0}$, and $n$ (usually called polytropic index) are constants. The quantity $f(\mathbf{x}, \mathbf{v}) d^{3} x d^{3} v$ denotes the number of stars with position and velocity vectors respectively within the elements $d^{3} x$ and $d^{3} v$ in position and 
velocity spaces. The polytropic distribution (24) exhibits, after an appropriate identification of the relevant parameters, the $q$-MaxEnt form. The entropic parameter $q$ can be expressed in terms of the index $n$ by identifying $n-3 / 2$ with $1 /(1-q)$ (see [30] for details; notice that here we are identifying $f$ with $p$, and not with the escort distribution $\left.\propto p^{q}\right)$. We obtain

$$
q=\frac{2 n-5}{2 n-3}
$$

The limit $n \rightarrow \infty$ (hence $q=1$ ) recovers the isothermal sphere case; for $n<5$ (hence $q<5 / 7$ ), finiteness for mass and energy naturally emerges. Polytropic distributions constitute the simplest, physically plausible models for self-gravitating stellar systems [32]. Alas, these models do not provide an accurate description of the observational data associated with real galaxies. In spite of this, the connection between the $S_{q}$ entropy and stellar polytropes is of considerable interest. Besides the (in our opinion) notable fact that, for a special range of values of $q$, non-extensive thermostatistics leads to finite stellar systems, the established connection between the $S_{q}$ entropy and stellar polytropic distributions is interesting for other reasons. Polytropic distributions arise in a very natural way from the theoretical study of self gravitating systems. The investigation of their properties has been of constant interest for theoretical astrophysicists during the last one hundred years [32]. Polytropic distributions are still the focus of an intense research activity [31], and the study of their basic properties constitutes a part of the standard syllabus of astrophysics students. Now, polytropic distributions happen to exhibit the form of q-MaxEnt distributions, that is, they constitute distribution functions in $(\mathbf{x}, \mathbf{v})$ space that maximize the non-extensive functional $S_{q}$ under the natural constraints imposed by the conservation of mass and energy [29] (other constraints associated with other conserved quantities may be incorporated). Let us recall that the original path leading to the $S_{q}$ entropic form was not motivated by self-gravitating systems, nor was it motivated by any other specific system. The entropic form $S_{q}$ was proposed by recourse to very general arguments dealing with the consideration of (i) entropic forms incorporating power law structures (inspired on multifractals) and reducing to the standard logarithmic measure in an appropriate limit and (ii) the basic properties a functional of 
the probabilities should have in order to represent a physically sensible entropic measure [21]. The $q$-entropy is a very natural and, to a certain extent, unique generalization of the Boltzmann-Gibbs-Shannon measure. Taking this into account, it is remarkable that the extremalization of the $q$-measure leads to a family of distribution functions of considerable importance in theoretical astrophysics. In a sense, we might say that astrophysicists, when studying newtonian self-gravitating systems, have been working with $q$-MaxEnt distribution functions for a hundred years without being aware of it. The discovery of the connection between $S_{q}$ and stellar polytropic distributions stimulated the application of nonextensive statistical mechanics to the study of other systems with long range interactions $[34,35]$. In particular, the analysis by Boghosian [34] of metastable states in pure electron plasmas lead to the identification of the first maximum $q$-entropy distribution observed in the laboratory $[34,35]$. In point of fact, the mathematical formalism used by Boghosian to describe the electron plasma is closely related to one associated to the stellar polytropic distributions [34].

Peculiar velocities of galaxy clusters

The COBE (Cosmic Background Explorer) satellite measured the peculiar velocities (difference of velocity with regard to the average expansion of the universe) of some clusters of spiral galaxies. A distribution was found which exhibits a cutoff around $500 \mathrm{Km} / \mathrm{s}$. The Princeton astrophysical group [36] analyzed the distribution of velocities within four different cosmological models (cold matter, hot matter, premieval barionic). None of those attempts succeeded in reproducing the observed cutoff, although each of those models involved several free parameters (that were fixed through a variety of arguments). By assuming within nonextensive statistical mechanics, an extremely - almost outrageously - simplified model, namely an ideal classical gas!, the empirical velocity distribution was quite satisfactorily matched [37]. Only two fitting parameters were used, namely the scale of velocities and $q \simeq 0.23 . \quad$ In spite of the extreme simplicity of the model, the fact that the statistics was allowed to change proved its high efficiency. The possible primacy of statistics over models is not new in statistical physics in fact. Even if we consider the ideal gas hypothesis 
for a quantum gas, we obtain acceptably good first approximations for phenomena such as superconductivity or suprafluidity (using Bose-Einstein statistics), or such as metallic conductivity of electrons (using Fermi-Dirac statistics). Of course, more realistic models must include interactions, but an acceptable first approach can indeed be done already on the basis of an appropriate statistics (even if the model is oversimplified).

\section{Cosmic rays:}

The flux of cosmic rays arriving on Earth is a quantity whose measured range is among the widest experimentally known (33 decades in fact). This distribution refers to a range of energies which also is impressive (13 decades). This distribution is very far from exponential, as can be verified on Fig 1. This basically indicates that no BG thermal equilibrium is achieved, but some other (either stationary, or relatively slow varying) state, characterized in fact by a power law. If the distribution is analyzed with more detail, one verifies that two, and not one, power-law regimes are involved, separated by what is called the "knee" (slightly below $10^{16} \mathrm{ev}$ ). At very high energies, the power-law seems to be interrupted by what is called the "ankle" (close to $10^{19} \mathrm{ev}$ ). One may guess that, along such wide ranges (of both fluxes and energies), a variety of complex intra- and inter-galactic phenomena are involved, related to both the sources of the cosmic rays as well the media they cross before arriving on Earth. However, from a phenomenological viewpoint, the overall results amount to something quite simple. Indeed, by solving a simple differential equation, a quite remarkable agreement is obtained [38]. This differential equation is the following one:

$$
\frac{d p_{i}}{d E_{i}}=-b^{\prime} p_{i}^{q^{\prime}}-b p_{i}^{q}
$$

This differential equation has remarkable particular cases. The most famous one is $\left(q^{\prime}, q\right)=$ $(1,2)$, since it precisely corresponds to the differential equation which enabled Planck, in his October 1900 paper, to (essentially) guess the black-body radiation distribution, thus opening (together with his December 1900 paper) the road to quantum mechanics. The more general case $q^{\prime}=1$ and arbitrary $q$ is a simple particular instance of the Bernoulli equation, and, as such, has a simple explicit solution. This solution has proved its efficiency in a variety 
of problems, including in generalizing the Zipf-Mandelbrot law for quantitative linguistics (see Montemurro's article in [23] for a review). Finally, the generic case $q>q^{\prime}>1$ also has an explicit solution (though not particularly simple, but in terms of two hypergeometric functions) and produces, taking also into account the ultra-relativistic ideal gas density of states, the above mentioned quite good agreement with the observed fluxes. Indeed, if we assume $0<b^{\prime}<<b$ and $q^{\prime}<q$, the distribution makes a crossover from a power-law characterized by $q$ at low energies to a power-law characterized by $q^{\prime}$ at high energies, which is exactly what the cosmic rays exhibit to a quite good approximation: See Fig. 1.

\section{Cosmology:}

Nonextensive statistical mechanics has also been applied to a variety of cosmological and general relativity problems including the cosmic background radiation in a RobertsonWalker universe, the dynamics of inflationary cosmologies, the universal density profile of dark halos, early universe phenomena (e.g., the primordial ${ }^{4} H e$ formation), among others $[39]$.

\section{CONCLUSIONS}

In outer space physics and astrophysics, there is a considerable amount of anomalous phenomena that require a thermostatistical treatment which exceeds the usual capabilities of Boltzmann-Gibbs statistical mechanics. This fact is due to the relevance of gravitational forces (which are long-ranged), as well as to a variety of dynamical nonlinear dynamical aspects. Some of these phenomena appear to be tractable within nonextensive statistical mechanics, and we have illustrated this with a few typical examples.

In addition to this, we have argued that, in analogy with the Ptolemy-Kepler problem concerning the orbits of the planets, the optimization of the nonextensive entropy $S_{q}$ with a few constraints can be equivalently seen as the optimization of the BG entropy with an infinite number of (ultimately related) constraints. The Keplerian orbit is equivalent to infinite Ptolemaic epicycles, and its series expansion in powers of the eccentricity $\epsilon$ abruptly 
reduces to a single cercle when $\epsilon$ precisely vanishes. Quite analogously, the variational problem associated with $S_{q}$ for the canonical distribution is equivalent to considering $S_{B G}$ with infinite (related) constraints, and its series expansion in powers of $q-1$ abruptly reduces to the $S_{B G}$ variational problem with a single constraint (besides the trivial one associated with the norm) when $q-1$ precisely vanishes

Last but not least, the statements by Fermi and by Tisza that we quoted in the Introduction constitute a reply which precisely points one of the various inadvertences contained in Nauenberg's recent criticism of nonextensive statistical mechanics [40]. The nonextensive thermostatistical formalism addresses, among others, conservative Hamiltonian many-body systems including long-range interactions. For such systems, the "surface energy" (using Fermi's expression), or the "interaction energy" (using Tisza's expression), is as relevant as the energies of the parts, and does not become negligible in the thermodynamic limit, in contrast with what happens for short-range interacting systems. Nauenberg violates precisely this by imposing his Eq. (7). Moreover, a few lines earlier (his Eq. (5)), he imposes the factorization of the probabilities. This property is inconsistent with his own Eq. (7). Indeed, the metaequibrium distribution of energies is given by the $q$-exponential function $e_{q}^{x} \equiv[1+(1-q) x]^{1 /(1-q)}$, and, unless $q=1$, generically $e_{q}^{x+y} \neq e_{q}^{x} e_{q}^{y}=e_{q}^{x+y+(1-q) x y}$. This type of inconsistency (for finite systems) has already been pointed out by several authors, starting with [41]. It is not impossible that this specific inconsistency disappears in the limit $N \rightarrow \infty$, if taken before the $t \rightarrow \infty$ limit, but this is highly nontrivial and remains to be proved.

\section{ACKNOWLEDGMENTS}

The possibility of interpreting the optimization of $S_{q}$ with a few constraints as an optimization of $S_{B G}$ with an infinite number of constraints emerged during a conversation between one of us (CT) and M. Gell-Mann, to whom we are very grateful. We are also in-

debted to A. Plastino, R.S. Mendes and E.P. Borges for useful remarks. This work has been 
partially supported by PRONEX/MCT, CNPq, and FAPERJ (Brazilian agencies). One of us (ARP) thanks financial support from MECyD (Spanish agency). 


\section{REFERENCES}

[1] E. Fermi, Thermodynamics (Dover, New York, 1956).

[2] L. Tisza, Generalized Thermodynamics (MIT Press, Cambridge, Massachussetts, 1966), page 123 .

[3] P.T. Landsberg, Thermodynamics and Statistical Mechanics (Oxford university Press, Oxford, 1978), page 102 [also Dover, New york, 1990].

[4] E.G.D. Cohen, Physica A 305, 19 (2002).

[5] M. Baranger, Physica A 305, 27 (2002).

[6] C. Beck, G. S. Lewis and H. L. Swinney, Phys. Rev. E 63, 035303 (2001); C. Beck, Phys. Rev. Lett. 87, 180601 (2001).

[7] T. Arimitsu and N. Arimitsu, Physica A 305, 218 (2002).

[8] I. Bediaga, E. M. F. Curado and J. Miranda, Physica A 286, 156 (2000).

[9] D.B. Walton and J. Rafelski, Phys. Rev. Lett. 84, 31 (2000).

[10] C. Anteneodo, C. Tsallis and A.S. Martinez, Europhys. Lett. 59, 635 (2002).

[11] L. Borland, Phys. Rev. Lett. 89, 098701 (2002); Quantitative Finance 2, 415 (2002).

[12] R. Osorio, L. Borland and C. Tsallis, in Nonextensive Entropy - Interdisciplinary Applications, M. Gell-Mann and C. Tsallis, eds. (Oxford University Press, 2003), in preparation; see also F. Michael and M.D. Johnson, Financial marked dynamics, Physica A (2003), in press.

[13] A. Upadhyaya, J.-P. Rieu, J.A. Glazier and Y. Sawada, Physica A 293, 549 (2001).

[14] J. A. S. de Lima, R. Silva and A. R. Plastino, Phys. Rev. Lett. 86, 2938 (2001).

[15] C. Tsallis, A.R. Plastino and W.-M. Zheng, Chaos, Solitons \& Fractals 8, 885 (1997);

U.M.S. Costa, M.L. Lyra, A.R. Plastino and C. Tsallis, Phys. Rev. E 56, 245 (1997); 
M.L. Lyra and C. Tsallis, Phys. Rev. Lett. 80, 53 (1998); U. Tirnakli, C. Tsallis and M.L. Lyra, Eur. Phys. J. B 11, 309 (1999); V. Latora, M. Baranger, A. Rapisarda, C. Tsallis, Phys. Lett. A 273, 97 (2000); F.A.B.F. de Moura, U. Tirnakli, M.L. Lyra, Phys. Rev. E 62, 6361 (2000); U. Tirnakli, G. F. J. Ananos, C. Tsallis, Phys. Lett. A 289, 51 (2001); H. P. de Oliveira, I. D. Soares and E. V. Tonini, Physica A 295, 348 (2001); F. Baldovin and A. Robledo, Europhys. Lett. 60, 518 (2002); F. Baldovin and A. Robledo, Phys. Rev. E 66, 045104(R) (2002); E.P. Borges, C. Tsallis, G.F.J. Ananos and P.M.C. Oliveira, Phys. Rev. Lett. 89, 254103 (2002); U. Tirnakli, Physica A 305, 119 (2002); U. Tirnakli, Phys. Rev. E 66, 066212 (2002).

[16] Y. Weinstein, S. Lloyd and C. Tsallis, Phys. Rev. Lett. 89, 214101 (2002).

[17] S. Abe and A.K. Rajagopal, Physica A 289, 157 (2001), C. Tsallis, S. Lloyd and M. Baranger, Phys. Rev. A 63; 042104 (2001); C. Tsallis, P.W. Lamberti and D. Prato, Physica A 295, 158 (2001); F.C. Alcaraz and C. Tsallis, Phys. Lett. A 301, 105 (2002); C. Tsallis, D. Prato and C. Anteneodo, Eur. Phys. J. B 29, 605 (2002); J. Batle, A.R. Plastino, M. Casas and A. Plastino, Conditional q-entropies and quantum separability: A numerical exploration, quant-ph/0207129 (2002).

[18] A.R. Plastino and A. Plastino, Physica A 222, 347 (1995); C. Tsallis and D.J. Bukman, Phys. Rev. E 54, R2197 (1996); C. Giordano, A.R. Plastino, M. Casas and A. Plastino, Eur. Phys. J. B 22, 361 (2001); A. Compte and D. Jou, J. Phys. A 29, 4321 (1996); A.R. Plastino, M. Casas and A. Plastino, Physica A 280, 289 (2000); M. Bologna, C. Tsallis and P. Grigolini, Phys. Rev. E 62, 2213 (2000); C. Tsallis and E.K. Lenzi, in Strange Kinetics, eds. R. Hilfer et al, Chem. Phys. 284, 341 (2002) [Erratum (2002)]; E.K. Lenzi, L.C. Malacarne, R.S. Mendes and I.T. Pedron, Anomalous diffusion, nonlinear fractional Fokker-Planck equation and solutions, Physica A (2003), in press [cond-mat/0208332]; E.K. Lenzi, C. Anteneodo and L. Borland, Phys. Rev. E 63, 051109 (2001); E.M.F. Curado and F.D. Nobre, Derivation of nolinear Fokker-Planck 
equations by means of approximations to the master equation, Phys. Rev. E 67, 0211XX (2003), in press; C. Anteneodo and C. Tsallis, Multiplicative noise: A mechanism leading to nonextensive statistical mechanics, cond-mat/0205314 (2002).

[19] C. Anteneodo and C. Tsallis, Phys. Rev. Lett 80, 5313 (1998); V. Latora, A. Rapisarda and C. Tsallis, Phys. Rev. E 64, 056134 (2001); A. Campa, A. Giansanti and D. Moroni, in Non Extensive Statistical Mechanics and Physical Applications, eds. G. Kaniadakis, M. Lissia and A. Rapisarda, Physica A 305, 137 (2002); B.J.C. Cabral and C. Tsallis, Phys. Rev. E 66, 065101(R) (2002); E.P. Borges and C. Tsallis, in Non Extensive Statistical Mechanics and Physical Applications, eds. G. Kaniadakis, M. Lissia and A. Rapisarda, Physica A 305, 148 (2002); A. Campa, A. Giansanti, D. Moroni and C. Tsallis, Phys. Lett. A 286, 251 (2001); M.-C. Firpo and S. Ruffo, J. Phys. A 34, L511 (2001); C. Anteneodo and R.O. Vallejos, Phys. Rev. E 65, 016210 (2002); R.O. Vallejos and C. Anteneodo, Phys. Rev. E 66, 021110 (2002); M.A. Montemurro, F. Tamarit and C. Anteneodo, Aging in an infinite-range Hamiltonian of coupled rotators, Phys. Rev. E (2003), in press [cond-mat/0205355].

[20] S. Abe and N. Suzuki, Phys. Rev. E 67, 016106 (2003).

[21] C. Tsallis, J. Stat. Phys. 52, 479 (1988).

[22] E.M.F. Curado and C. Tsallis, J. Phys. A: Math. Gen. 24, L69 (1991) [Corrigenda: 24, 3187 (1991) and 25, 1019 (1992)]; C. Tsallis, R.S. Mendes and A.R. Plastino, Physica A 261, 534 (1998).

[23] S.R.A. Salinas and C. Tsallis, eds., Nonextensive Statistical Mechanics and Thermodynamics, Braz. J. Phys. 29, No. 1 (1999); S. Abe and Y. Okamoto, eds., Nonextensive Statistical Mechanics and its Applications, Series Lecture Notes in Physics (SpringerVerlag, Berlin, 2001); G. Kaniadakis, M. Lissia and A. Rapisarda, eds., Non Extensive Statistical Mechanics and Physical Applications, Physica A 305, No 1/2 (Elsevier, Amsterdam, 2002); M. Gell-Mann and C. Tsallis, eds., Nonextensive Entropy - Interdis- 
ciplinary Applications (Oxford University Press, 2003), in preparation; H.L. Swinney and C. Tsallis, eds., Anomalous Distributions, Nonlinear Dynamics, and Nonextensivity, Physica D (2003), in preparation. An updated bibliography can be found at the web site http://tsallis.cat.cbpf.br/biblio.htm

[24] J. Henry Moving Heavens and Earth: Copernicus and the Solar System (Icon Books, Cambridge, 2001).

[25] F. Hoyle, Nicolaus Copernicus - An essay on his life and work (Harper and Row, Publishers, New York, 1973), page 14.

[26] J.M.A. Danby, Fundamentals of Celestial Mechanics, Second Edition, (Willmann-Bell Inc., Richmond, 1989).

[27] F.A.B.F. de Moura, U. Tirnakli and M.L. Lyra, Phys. Rev. E 62, 6361 (2000).

[28] G. Kaniadakis, A. Lavagno and P. Quarati, Phys. Lett. B 369, 308 (1996); P. Quarati, A. Carbone, G. Gervino, G. Kaniadakis, A. Lavagno and E. Miraldi, Nucl. Phys. A 621, 345c (1997); G. Kaniadakis, A. Lavagno and P. Quarati, Astrophysics and space science 258, 145 (1998); G. Kaniadakis, A. Lavagno, M. Lissia and P. Quarati, in Proc. 5th International Workshop on Relativistic Aspects of Nuclear Physics (Rio de JaneiroBrazil, 1997); eds. T. Kodama, C.E. Aguiar, S.B. Duarte, Y. Hama, G. Odyniec and H. Strobele (World Scientific, Singapore, 1998), p. 193; G. Gervino, G. Kaniadakis, A. Lavagno, M. Lissia and P. Quarati, Non-markovian effects in the solar neutrino problem, communicated at "Nuclei in the Cosmos" (Volos-Greece, July 1998); M. Coraddu, G. Kaniadakis, A. Lavagno, M. Lissia, G. Mezzorani and P. Quarti, in Nonextensive Statistical Mechanics and Thermodynamics, eds. S.R.A. Salinas and C. Tsallis, Braz. J. Phys. 29, 153 (1999); A. Lavagno and P. Quarati, Nucl. Phys. B, Proc. Suppl. 87, 209 (2000); C.M. Cossu, Neutrini solari e statistica di Tsallis, Master Thesis, Universita degli Studi di Cagliari (2000). 
[29] A. R. Plastino and A. Plastino, Physics Letters A 174, 384 (1993).

[30] A.R. Plastino, in Nonextensive Statistical Mechanics and Its Applications, eds. S. Abe and Y. Okamoto (Springer, Berlin, 2001).

[31] J.-J. Aly and J. Perez, Phys. Rev. E 60, 5185 (1999); A. Taruya and M. Sakagami, Physica A 307, 185 (2002); A. Taruya and M. Sakagami, Gravothermal catastrophe and Tsallis' generalized entropy of self-gravitating systems II. Thermodynamic properties of stellar polytrope, Physica A (2003), in press [cond-mat/0204315]; P.-H. Chavanis, Astro. and Astrophys. 386, 732 (2002); P.H. Chavanis, Gravitational instability of isothermal and polytropic spheres, Astro. and Astrophys. (2003), in press [astro-ph/0207080].

[32] J. Binney and S. Tremaine, Galactic dynamics (Princeton University Press, Princeton, 1987).

[33] S. Tremaine, M. Hénon and D. Lynden-Bell, Mon. Not. R. Astron. Soc. 219, 285 (1986).

[34] B. M. Boghosian, Phys. Rev. E, 53, 4754 (1996).

[35] C. Anteneodo and C. Tsallis, J. Mol. Liq. 71, 255 (1997).

[36] N.A. Bahcall and S.P. Oh, Ap. J. Lett. 462, L49 (1996).

[37] A. Lavagno, G. Kaniadakis, M. Rego-Monteiro, P. Quarati and C. Tsallis, Astrophysical Letters and Communications 35, 449 (1998).

[38] C. Tsallis, J.C. Anjos and E.P. Borges, Fluxes of cosmic rays: A delicately balanced anomalous stationary state, astro-ph/0203258 (2002).

[39] V.H. Hamity and D.E. Barraco, Phys. Rev. Lett. 76, 4664 (1996); V.H. Hamity and D.E. Barraco, Physica A 282, 203 (2000); L.P. Chimento, J. Math. Phys. 38, 2565 (1997); D.F. Torres, H. Vucetich and A. Plastino, Phys. Rev. Lett. 79, 1588 (1997) [Erratum: 80, 3889 (1998)]; U. Tirnakli and D.F. Torres, Physica A 268, 225 (1999); L.P. Chimento, F. Pennini and A. Plastino, Physica A 256, 197 (1998); L.P. Chimento, 
F. Pennini and A. Plastino, Phys. Lett. A 257, 275 (1999); D.F. Torres and H. Vucetich, Physica A 259, 397 (1998); D.F. Torres, Physica A 261, 512 (1998); H.P. de Oliveira, S.L. Sautu, I.D. Soares and E.V. Tonini, Phys. Rev. D 60, 121301-1 (1999); M.E. Pessah, D.F. Torres and H. Vucetich, Physica A 297, 164 (2001); M.E. Pessah and D.F. Torres, Physica A 297, 201 (2001); C. Hanyu and A. Habe, Astrophys. J. 554, 1268 (2001); E.V. Tonini, Caos e universalidade em modelos cosmologicos com pontos criticos centro-sela, Doctor Thesis (Centro Brasileiro de Pesquisas Fisicas, Rio de Janeiro, March 2002).

[40] M. Nauenberg, A critique of non-extensive q-entropy for thermal statistics, condmat/0210561 (2002) [version 2].

[41] G.R. Guerberoff and G.A. Raggio, J. Math. Phys. 37, 1776 (1996); G.R. Guerberoff, P.A. Pury and G.A. Raggio, J. Math. Phys. 37, 1790 (1996). 


\section{FIGURES}

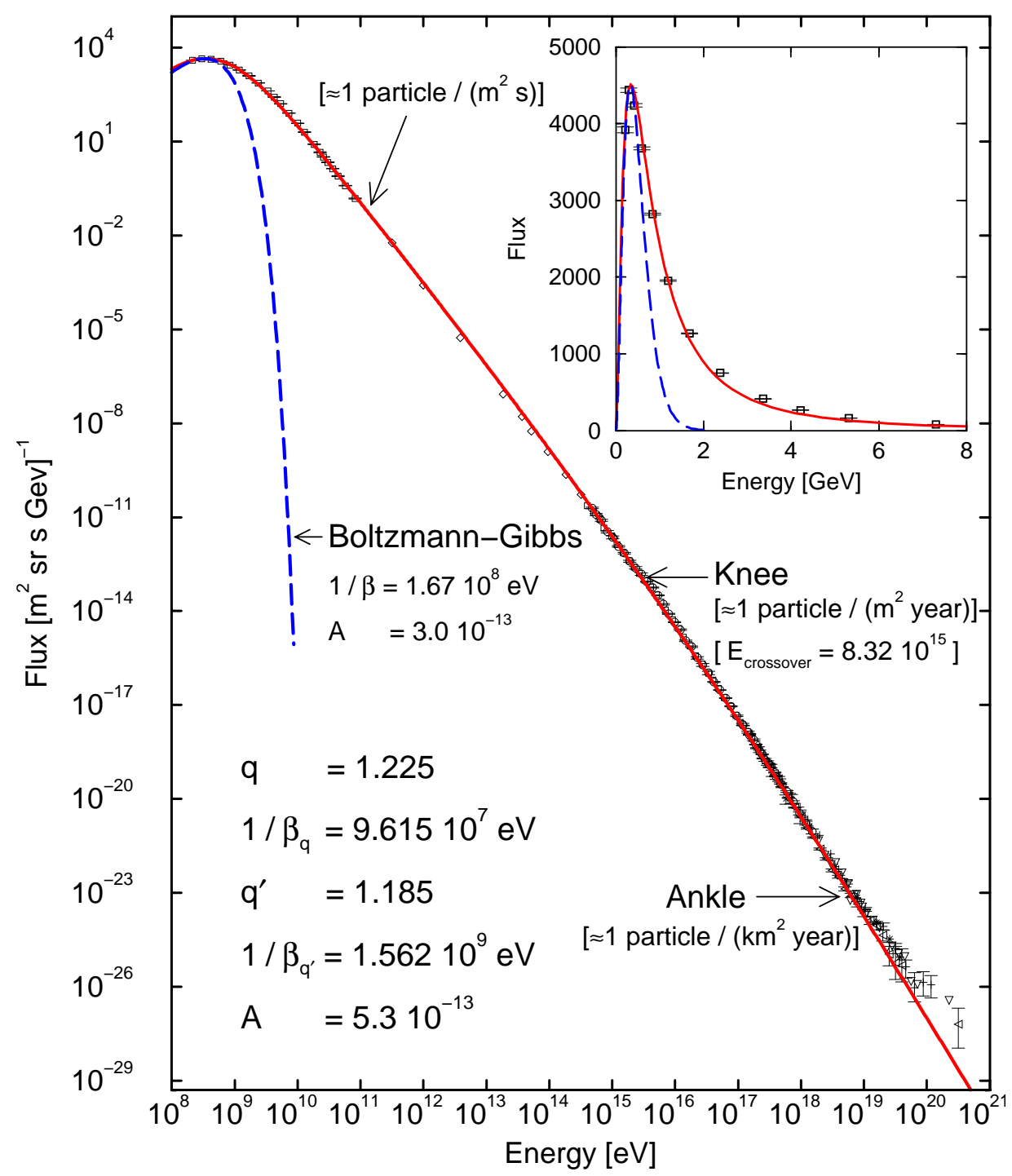

FIG. 1. Flux of cosmic rays as a function of their energies. See [38] for the origin of the experimental data and theoretical details. The blue dashed curve corresponds to the BG distribution, and the red continuous curve to a crossover between a $q=1.225$ distribution (before the $k n e e$ ) and a $q^{\prime}=1.185$ one (after the knee). 